\title{
Mutations in the $\alpha$-helix directly $C$-terminal to the major homology region of human immunodeficiency virus type 1 capsid protein disrupt Gag multimerization and markedly impair virus particle production
}

\author{
Hung-Hao Chu ${ }^{1,2}$, Yu-Fen Chang ${ }^{1,2} \&$ Chin-Tien Wang ${ }^{1,2, *}$ \\ ${ }^{1}$ Department of Medical Research and Education, Taipei Veterans General Hospital, 201, Sec. 2, Shih-Pai \\ Road, Taipei, 11217, Taiwan; ${ }^{2}$ Institute of Clinical Medicine, National Yang-Ming University School of \\ Medicine, Taipei, Taiwan
}

Received 20 March 2006; accepted 20 May 2006

(c) 2006 National Science Council, Taipei

Key words: Gag, HIV-1, major homology region, virus assembly

\begin{abstract}
The X-ray crystallographic structure of HIV-1 capsid protein suggests that the dimer interface of the dimerization domain is mainly formed from a putative $\alpha$-helix structure of 14 amino acids (Gag residues 311-324) and lies directly C-terminal to the capsid major homology region. We found that a deletion mutation in the $\alpha$-helix drastically reduces virus particle production. Alanine-scanning mutagenetic analysis indicated that substitution mutations at residues Q311, V313, K314, W316, and M317 all impair virus particle production markedly. Membrane flotation assays suggested that some mutations in the dimer interface have slight effects on the efficient binding of Gag to membranes. Indirect immunofluorescence studies revealed that mutants defective in virus production exhibit a subcellular distribution pattern similar to that of wild-type. However, velocity sedimentation analysis showed that mutations significantly impairing virus particle production were also detrimental to Gag multimerization, suggesting that the impaired virus production may be due to a defect in Gag multimerization. These results support the proposal that residues in the capsid dimer interface play a crucial role in promoting Gag multimerization, possibly by facilitating stable Gag-Gag interactions.
\end{abstract}

\section{Introduction}

The major structural protein of all retroviruses, including human immunodeficiency virus (HIV), is encoded by gag [reviews, 1-3]. In HIV, the Gag sequence is synthesized initially as a polyprotein precursor, $\operatorname{Pr} 55^{\text {gag }}$. During or after virus budding, $\operatorname{Pr} 55^{\text {gag }}$ is proteolytically processed by the viral protease (PR) into four major products: matrix (MA; p17), capsid (CA; p24), nucleocapsid (NC; p7), and the C-terminal p6 [4-7]. In addition, there are two small spacer peptides termed SP1 and SP2 which separate $\mathrm{NC}$ from $\mathrm{CA}$ and $\mathrm{p} 6$, respectively.

*To whom correspondence should be addressed. Fax: + 886-228742279; E-mail: chintien@ym.edu.tw
The PR-mediated processing of virus particles is not required for virus particle assembly, but is essential for virus infectivity [8-11]. Besides PR, the enzymes required for virus replication are also encoded by pol. These include the reverse transcriptase (RT), RNase H, and integrase (IN). The HIV-1 Pol sequence is synthesized as a Gag-Pol polyprotein [12] and is incorporated into virions by interaction with $\operatorname{Pr} 55^{\text {gag }}$ through its $\mathrm{N}$-terminal Gag domain [13-16].

In the absence of other viral components, expression of $g a g$ alone is sufficient for production of virus-like particles, which suggests that the Gag precursor contains the information necessary to direct virus particle assembly and budding $[17,18]$. 
Functions involving Gag transport, assembly, and budding have been ascribed to specific domains, referred to as the membrane binding $(\mathrm{M})$, interaction (I), or late assembly (L) domains. In HIV-1, the $\mathrm{M}$ domain, located at the $\mathrm{N}$-terminal end of MA, consists of a co-translationally modified myristylation signal and a cluster of basic residues. Mutations at the $\mathrm{M}$ domain disrupt membrane association and plasma membrane targeting of Gag, resulting in disruption of virus particle production [19-22]. The I domain, thought to be responsible for Gag-Gag interaction, mainly lies in NC. NC mutations result in reduction of virus particle production and affect virus particle density, presumably by interfering with proper Gag packaging [23-26]. In addition to NC, HIV-1 CA is also functionally involved in Gag-Gag interactions that are required for virus assembly [27-30]. The HIV-1 L domain containing the PT/SAP motif lies in the p6. Removal of $\mathrm{p} 6$ or mutations in the L domain do not affect virus particle assembly significantly, but markedly block the release of virions from the cell membrane [review, 31].

A number of studies have shown that the gag coding sequence can be extensively deleted without significantly affecting virus-like particle assembly and budding [32-34]. HIV gag mutants lacking the sequence upstream of the CA major homology region (MHR), but retaining the N-terminal myristylation signal and the $\mathrm{C}$-terminal $\mathrm{L}$ domain, could still produce virus particles efficiently [35]. These data suggest that the C-terminal one-third of the gag sequence contains domains necessary for Gag assembly and release. There is an increasing amount of published data on mapping of the domains responsible for Gag-Gag interactions, and such studies have implicated the C-terminal $\mathrm{CA}$ and the downstream NC [36-39]. In particular, it has been shown that deletion or some substitution mutations in MHR markedly reduce virus particle production [27, 38, 40]. Several recent studies have shown that the extreme C-terminal sequence of CA and also SP1 play a key role in the process of Gag multimerization and virus assembly in HIV [36, 39, 41, 42]. In a previous study, we found that a 14-codon deletion directly C-terminal to MHR not only markedly reduced virus particle production, but also significantly affected the incorporation of HIV-1 Gag-Pol into wild-type Gag particles [43], suggesting that this region may be functionally involved in both $\mathrm{Gag} / \mathrm{Gag}$ and
Gag-Pol/Gag interaction. Crystallography has revealed that the dimer interface of the $\mathrm{C}$-terminal dimerization domain is formed by a putative $\alpha$-helix, which lies adjacent to the C-terminus of MHR and consists of 14 amino acid residues [28, 44]. Point mutations in this region have been reported to reduce virus particle production and block CA dimerization in vitro [28], indicating that the $\alpha$-helix contributes to Gag-Gag interaction during virus assembly. We extended the previous study to elucidate the role of the $\alpha$-helix in the process of virus assembly. Our results indicate that substitution mutations in this region can disrupt virus assembly without significantly affecting the subcellular distribution of Gag. However, the oligomerization of Gag into high molecular weight complexes is markedly affected by the mutations, suggesting that the $\alpha$-helix directly $\mathrm{C}$-terminal to the capsid MHR is required for efficient Gag multimerization.

\section{Methods}

\section{Plasmid construction}

The HIV gag deletion mutants were derived from recombination of BamHI-containing constructs. Initially, a number of BamHI sites were created at the following nucleotide positions by PCR-mediated mutagenesis: 1715, 1758, 1802, 1884 and 1923. Recombination of the BamHI linker constructs 1715 and 1758, 1758 and 1802, 1802 and 1884,1884 and 1923 yielded constructs $\Delta 310-323$, $\Delta 322-338, \Delta 339-365$, and $\Delta 368-378$, respectively (Figure 1). The $\Delta 281-308$ mutation, with a deletion of the whole CA major homology region (MHR), has been described previously [13]. To construct alanine substitution mutants S310A, Q311A, E312A, V313A, K314A, N315A, W316A, M317A, T318A, and E319A, DNA fragments containing the mutations were first generated by each of the mutation-containing primers (Table 1) and a reverse primer, 2577-51 (5'-ACT GGTACAGTCTCAATAGGGCTAATG-3'), using HIVgpt [45] as template. Each of the resulting PCR products was then used as a megaprimer for a second round of PCR by using the forward primer G80 (5'-ATGAGAGAACCAAGGGGAAGTGT GA-3'). The PCR products were then digested with Spe1 and BclI and ligated into HIVgpt. Each 


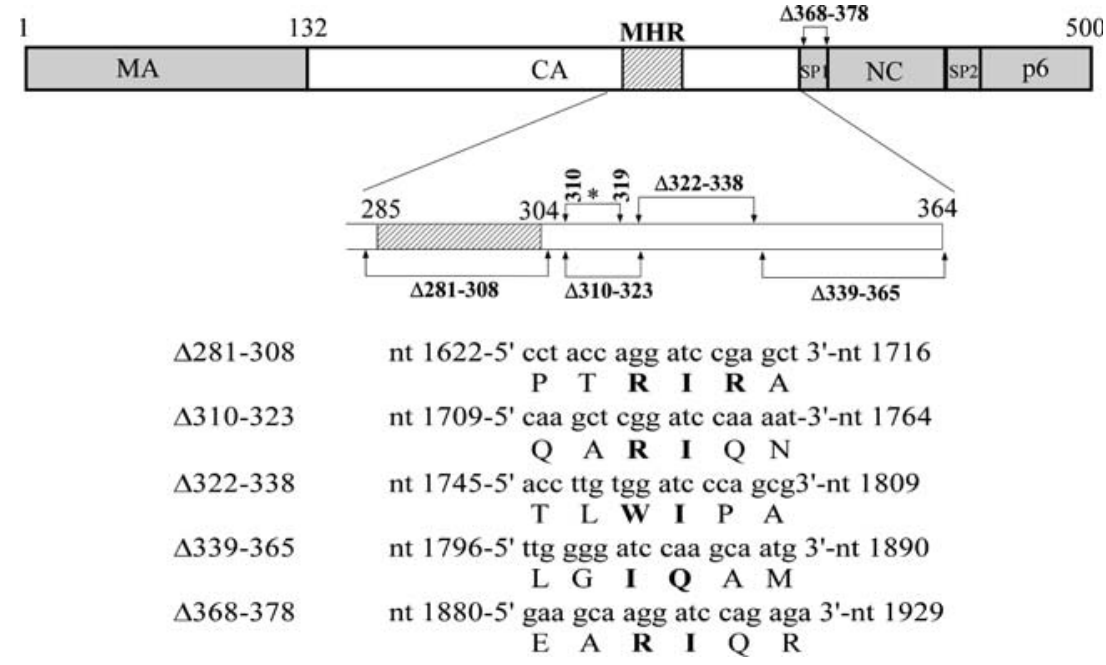

Figure 1. Schematic representations of wild-type and mutant HIV-1 expression constructs. The wild-type (WT) Gag protein domains MA (matrix; p17), CA (capsid, p24), major homology region (MHR), NC (nucleocapsid, p7) and p6; and the two small spacer peptides SP1 and SP2 are indicated. The numbers indicate the Gag amino acid residue positions, and are used to denote each of the mutant constructs with a deletion mutation in Gag. The boundaries of deleted Gag sequences are indicated (solid lines and arrowheads). Asterisk $(*)$ denotes the point mutation with alanine substitution at residues $310-319$. The sequences and the altered amino acid residues (boldface) in the mutated region are given.

of the mutant constructs was analyzed by restriction enzyme digestion and its identity confirmed by DNA sequencing. The myristylation-minus $\left(\mathrm{Myr}^{-}\right)$mutation, in which the second glycine residue has been replaced by alanine, blocks Gag membrane binding and virus production [46].

Table 1. Nucleotide sequences of the primers used to create the mutations.

\begin{tabular}{lll}
\hline Mutations & $\begin{array}{l}\text { Oligodeoxynucleotide } \\
\text { sequence (sense) }\end{array}$ & $\begin{array}{l}\text { Altered } \\
\text { restriction } \\
\text { sites }\end{array}$ \\
\hline S310A & $5^{\prime}$-gagc aagctgcacaggaggta-3' & HindIII(-) \\
Q311A & $5^{\prime}$-gagcaagctt cagctgaggtaaaaaat-3' & PvuII \\
E312A & $5^{\prime}$-gagc aagcatcacaggeggtaaaaaat-3' & HindIII(-) \\
V313A & $5^{\prime}$-caa gctagccaggaggcaaaaaattg-3' & NheI \\
K314A & $5^{\prime}$-tcacaggagg tggccaattggatgaca-3' & MscI \\
N315A & $5^{\prime}$-c aagcatcacaggaggtaaaagcat & HindIII(-) \\
& ggatg-3' & \\
W316A & $5^{\prime}$-aaaaatgagatgacaga aacgttgttg-3' & AclI \\
M317A & $5^{\prime}$-taaaaaattgggegacaga aacgttgttg-3' & AclI \\
T318A & $5^{\prime}$-tggatggcaga aacgttgttggtc-3' & AclI \\
E319A & $5^{\prime}$-tggatgacagc aacgttgttggtc-3' & AclI \\
\hline
\end{tabular}

Nucleotides altered to create the alanine substitution mutants are shown in boldface. The nucleotides underlined indicate a silent mutation that creates a new restriction site or destroys the original one (-).
Each of thegag mutations was also introduced into an HIV-1 Pr55 gag expression vector, pGAG [47], yielding a set of PR-defective versions

\section{Cell culture and transfection}

$293 \mathrm{~T}$ cells and HeLa cells were maintained in DMEM supplemented with $10 \%$ fetal calf serum. Confluent 293T cells were trypsinized, split 1:10 and seeded onto $10-\mathrm{cm}$ plates $24 \mathrm{~h}$ before transfection. For each construct, 293T cells were transfected with $20 \mu \mathrm{g}$ of plasmid DNA by the calcium phosphate precipitation method [48], with the addition of $50 \mu \mathrm{m}$ chloroquine to enhance transfection efficiency. For transfection of HeLa cells, plasmid DNAs were mixed with lipofectamine 2000 (Invitrogen) at a ratio of $1(\mu \mathrm{g})$ : $2.5(\mu 1)$, and the transfection procedure followed the manufacturer's protocol.

\section{Western immunoblot analysis}

Culture media from transfected $293 \mathrm{~T}$ cells were filtered through $0.45-\mu \mathrm{m}$ pore-size filters, followed by centrifugation through $2 \mathrm{ml}$ of $20 \%$ sucrose in TSE (10 mM Tris-HCl, pH 7.5, $100 \mathrm{mM} \mathrm{NaCl}$, $1 \mathrm{mM}$ EDTA) containing $0.1 \mathrm{mM}$ phenylmethylsulfonyl fluoride (PMSF) at $4^{\circ} \mathrm{C}$ for $40 \mathrm{~min}$ at 
$274,000 \times g($ SW41 rotor at 40,000 rpm $)$. The viral pellets were then suspended in IPB $(20 \mathrm{mM}$ Tris$\mathrm{HCl}, \mathrm{pH} 7.5,150 \mathrm{mM} \mathrm{NaCl}, 1 \mathrm{mM}$ EDTA, $0.1 \%$ SDS, $0.5 \%$ sodium deoxycholate, $1 \%$ Triton X-100, $0.02 \%$ sodium azide) containing $0.1 \mathrm{mM}$ PMSF. The cells were rinsed with ice-cold phosphate-buffered saline (PBS), scraped from the plates, collected in $1 \mathrm{ml}$ PBS, and pelleted at $2,500 \mathrm{rpm}$ for $5 \mathrm{~min}$. The cell pellets were resuspended in $250 \mu \mathrm{l}$ of IPB containing $0.1 \mathrm{mM}$ $\mathrm{PMSF}$, and then subjected to microcentrifugation at $4{ }^{\circ} \mathrm{C}$ for $15 \mathrm{~min}$ at $13,700 \times g$ to remove cell debris. The supernatant and cell samples were mixed with equal volumes of $2 \times$ sample buffer (12.5 mM Tris- $\mathrm{HCl}$, pH 6.8, 2\% SDS, 20\% glycerol, $0.25 \%$ bromophenol blue) containing $\beta$-mercaptoethanol $(5 \%)$, and boiled for $5 \mathrm{~min}$. Samples were subjected to SDS-PAGE and electroblotted onto nitrocellulose membranes. The membranes were blocked with $3 \%$ gelatin in Tris-buffered saline containing $0.05 \%$ Tween 20 (TBST), followed by incubation with the primary antibody in $1 \%$ gelatin-TBST for $1 \mathrm{~h}$ on a rocking platform at room temperature. The membranes were then washed three times for $10 \mathrm{~min}$ each with TBST, and rocked for 30 min with the secondary antibody in $1 \%$ gelatin-TBST. The blots were again washed three times in TBST for 10 min each, and the membrane-bound antibody-conjugated enzyme activity was detected by an enhanced chemiluminescence (ECL) detection system or by a colorimetric method. For detection of HIV Gag proteins, we used an anti-p24 $4^{\text {gag }}$ monoclonal antibody (mouse hybridoma clone 183-H12-5C) at a dilution of 1:5,000 from ascites, or an antiHIV-1 p24 monoclonal antibody (NEA-9306, NEN Life Science Products Inc.) diluted 1:2,000. The secondary antibody was either a horse antimouse IgG alkaline phosphatase conjugate at 1:5,000 dilution (Vector Laboratories) or a sheep anti-mouse horseradish peroxidase- (HRP)-conjugated antibody at 1:15,000 dilution. The Gag proteins were visualized with a color reaction solution of NBT/BCIP (Promega), or with an enhanced chemiluminescence (ECL) kit according to the manufacturer's protocol (Pierce).

\section{Indirect immunofluorescence}

The protocol used for immunofluorescence was as described previously [34]. Briefly, confluent $\mathrm{HeLa}$ cells were split 1:80 and seeded onto coverslips $24 \mathrm{~h}$ before transfection. Two days after transfection, the cells were fixed at $4^{\circ} \mathrm{C}$ for $20 \mathrm{~min}$ in icecold PBS containing 3.7\% formaldehyde. They were then washed once with PBS and once with DMEM containing 10\% heat-inactivated calf serum (DMEM/calf serum) and permeabilized at room temperature for $10 \mathrm{~min}$ in PBS containing $0.2 \%$ Triton $\mathrm{X}-100$. The samples were incubated with primary antibodies for $1 \mathrm{~h}$ and with secondary antibodies for $30 \mathrm{~min}$. Following each incubation, the slides were subjected to three 5- to 10-min washes with DMEM/calf serum. The primary antibody was a mouse anti-HIV-1 CA monoclonal antibody at 1:500 dilution, and the secondary antibody was a rabbit anti-mouse rhodamine-conjugated antibody at 1:100 dilution (Cappel). After the last DMEM/calf serum wash, the coverslips were washed three times with PBS and mounted in $50 \%$ glycerol in PBS for viewing. Images were taken using an Olympus AX-80 fluorescence microscope.

\section{Membrane flotation assays}

At $48 \mathrm{~h}$ posttransfection, 293T cells were rinsed twice, pelleted in PBS, and resuspended in TE buffer (10 mM Tris-HCl, pH 7.4, 1 mM EDTA) containing $10 \%$ sucrose, and Complete protease inhibitor cocktail (Roche). Cell suspensions then were homogenized in a Wheaton dounce type A pestle followed by microcentrifugation at $3,000 \mathrm{rpm}$ for $5 \mathrm{~min}$ at $4^{\circ} \mathrm{C}$ to remove nuclei and cell debris. The postnuclear supernatants collected were subjected to ultracentrifugation at $55,000 \mathrm{rpm}$ for $15 \mathrm{~min}$ at $4^{\circ} \mathrm{C}$ (TLS-55 rotor, Beckman). The pellets then were suspended in TEN buffer (50 mM Tris-HCl, pH 7.4, $2 \mathrm{mM}$ EDTA, $150 \mathrm{mM} \mathrm{NaCl}$ ) containing $10 \%$ sucrose and Complete protease inhibitor cocktail. Two hundred $\mu \mathrm{l}$ of the suspension was mixed with $1.3 \mathrm{ml}$ of $87.5 \%$ sucrose in TEN buffer and placed at the bottom of a centrifuge tube. Sucrose solutions of $7 \mathrm{ml} 65 \%$ sucrose and $1.5 \mathrm{ml} 10 \%$ sucrose in $\mathrm{TN}$ buffer were then layered on top of this $1.5-\mathrm{ml}$ mixture. The gradients were centrifuged at $100,000 \times g$ for $16-18 \mathrm{~h}$ at $4^{\circ} \mathrm{C}$. Ten $1-\mathrm{ml}$ fractions were collected from the top of the centrifuge tube. The proteins in each of the fractions were precipitated with ice-cold 10\% 
trichloroacetic acid (TCA) and analyzed by western immunoblot.

\section{Velocity sedimentation analysis of cytoplasmic Gag proteins}

We adopted the protocol of Melamed et al. [39] to analyze the cytoplasmic Gag complex. Briefly, cells were rinsed twice with PBS, pelleted and resuspended in $1 \mathrm{ml}$ TEN buffer containing Complete protease inhibitor cocktail followed by homogenization as described above. The cell lysates then were centrifuged at $3000 \mathrm{rpm}$ for $20 \mathrm{~min}$ at $4{ }^{\circ} \mathrm{C}$. Five hundred microliter of the postnuclear supernatants were mixed with an equal amount of TEN buffer, and were then applied to the top of a premade $25-45 \%$ discontinuous sucrose gradient. This gradient was prepared in TEN buffer containing $1 \mathrm{ml}$ of each of $25 \%, 35 \%$, and $45 \%$ sucrose. The gradient was then centrifuged at $130,000 \times g$ for $1 \mathrm{~h}$ at $4^{\circ} \mathrm{C}$. Four $1-\mathrm{ml}$ fractions were collected from the top of the centrifuge tubes. The proteins present in aliquots of each fraction were precipitated with $10 \%$ TCA and subjected to western blot analysis as described in the membrane flotation assay.

\section{Results}

Deletion or substitution mutations in the $\alpha$-helix directly $C$-terminal to the $C A M H R$ diminish virus particle production markedly

We have found previously that a deletion of 14 amino acid residues ( $\Delta 310-323)$ immediately $\mathrm{C}$-terminal to the major homology region (MHR) of HIV-1 CA dramatically blocks virus assembly, and it also markedly affects the incorporation of Gag-Pol into Gag particles when introduced into an HIV-1 Gag-Pol expression vector [43]. This suggests that the sequence spanning the 14 amino acid residues may be functionally involved in the process of virus assembly. Since the C-terminal CA domain including the MHR is the domain primarily responsible for Gag assembly, we initially constructed a number of C-terminal CA deletion mutations ( $\Delta 281-308$, $\Delta 322-338$, and $\Delta 339-365$; Figure 1 ), and compared the effect of these mutations on virus assembly with that of $\Delta 310-323$. Because the sequence covering the CA-SP1 boundary has recently been reported to play a crucial role in virus assembly [32, 41, 42], a construct with deletion of the SP1 domain $(\Delta 368-378)$ was also included in this study.

Wild-type (wt) and mutant constructs were transiently expressed in $293 \mathrm{~T}$ cells, and virus particle assembly and processing were analyzed by western immunoblot. The results shown in Figure 2 suggest that all the mutations affected virus assembly, in accordance with the proposal that the C-terminal CA domain is critical for virus assembly. Mutations $\Delta 310-323, \Delta 281-308$, and $\Delta 368-378$ appear to be more detrimental to virus production than mutations $\Delta 322-338$ and $\Delta 339-365$. Most of the mutants were defective in particle processing, as substantial quantities of Gag remained in unprocessed or partially processed forms (Figure 2A, lanes 2-5).

Since the MHR is well-known for its importance in virus assembly, and since the roles of the C-terminal sequence of CA and the CA-SP1 boundary in virus assembly have been both well established, we concentrated on elucidating the role of the Gag residues 310-324 in the process of HIV assembly. To this end, we employed alaninescanning mutagenetic analysis. Each of the Gag residues from 310 to 319 was individually replaced with alanine (Figure 1), and the resultant mutants were examined for their ability to assemble and process virus particles. The results shown in Figure 3 indicate that all of the mutants had a steady-state expression level comparable to that of wt; however, some of the mutations had moderately to significantly impaired virus particle production. In particular, mutants Q311A, K314A, and W316A were markedly defective in virus production, all producing virions at a level below $30 \%$ of that of wt. This suggests that residues Q311, K314, and W316 are critical for virus particle production. Compared to the processing profile of $\mathrm{wt}$, where the mature $\mathrm{p} 24^{\text {gag }}$ represents the major species of virus-associated Gag, mutants Q311A and M317A both had substantial amounts of Gag unprocessed or incompletely processed (Figure 3A, lanes 4 and 11; Table 2). To test whether PR activity has any effect on virus particle production, all the mutants were expressed in a PR-defective version. We found that the level of virus-associated Gag precursor for each of the mutants was not significantly increased (data not 
Cell lysates
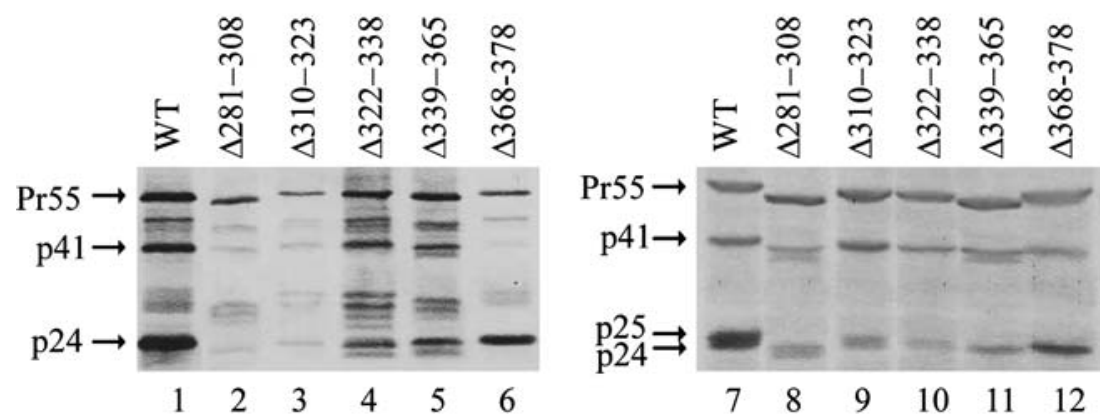

B

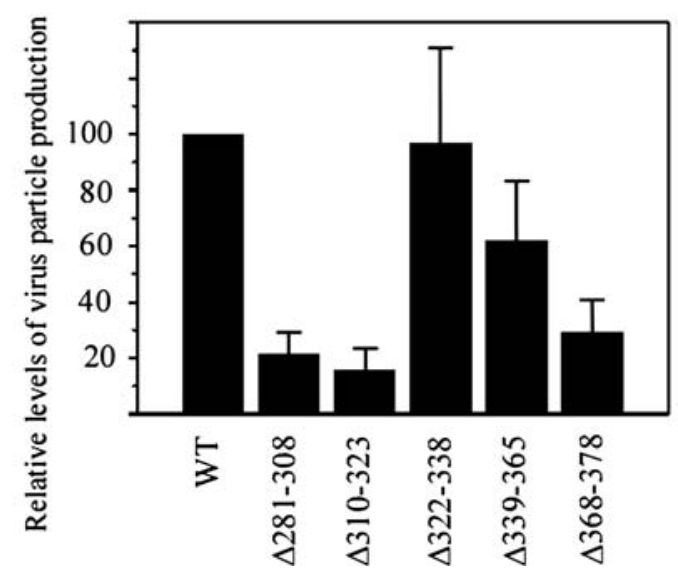

Figure 2. Assembly and processing of wild-type and mutant HIV Gag proteins. (A) 293T cells were transfected with $20 \mu \mathrm{g}$ of the designated plasmid. At $48 \mathrm{~h}$ posttransfection, culture supernatants and cells were collected and prepared for protein analysis as described in Materials and methods. Viral pellet samples (lanes 1-6) corresponding to $50 \%$ of the total samples, and cell lysate samples (lanes 7-12) corresponding to $5 \%$ of the total samples, were fractionated by SDS-PAGE (10\%) and electroblotted onto a nitrocellulose filter. HIV Gag proteins were probed with a mouse monoclonal antibody directed against the N-terminal part of CA $\left(\mathrm{p} 24^{g a g}\right)$. The positions of HIV Gag proteins Pr55, p41, p24/25, and p24 are indicated on the left. (B) Relative levels of virus particle production. Virus-associated Gag proteins were quantified by scanning mutant and wild-type p2 $4^{\text {gag }}$-associated band densities from immunoblots. The total arbitrary densitometer units of each mutant were normalized to that of the wild type in parallel experiments. Values of the ratios indicate the relative levels of virus particles released. Error bars indicate standard deviation.

shown), suggesting that the defect in virus particle production is independent of the PR-mediated particle processing

\section{Most of the mutations reducing virus particle production have no significant effects on the membrane binding and subcellular distribution of $\mathrm{Gag}$}

Because membrane association is a prerequisite for HIV Gag assembly and budding, and because point mutations in the MHR [49] or in the CA-SP1 boundary have been reported to diminish membrane binding of Gag [37, 41, 42], we performed membrane flotation assay to assess the membrane binding capacity of our mutants. To distinguish membrane-associated Gag from free cytoplasmic oligomerized Gag, crude membrane pellets were prepared and subjected to membrane flotation as described in Methods. Band densities in fractions 1-3 were divided by the total band densities of factions $1-10$ and multiplied by 100 to obtain percentage of membrane-bound protein for each construct (Figure 4). Analysis indicates that over $90 \%$ of the pelletable S310A, Q311A, and M317A were membrane-associated, a result that is comparable to that of wt. In contrast, less than $5 \%$ of the myristylation-minus (myr-) Gag, which has been shown to be defective in membrane binding [46], is membrane-associated. The $\Delta 310-323$ 
Table 2. Processing of HIV-1 Gag mutants.

\begin{tabular}{lllll}
\hline & WT & S310A & Q311A & E312A \\
Pr55 & $26 \pm 1$ & $24 \pm 1$ & $40 \pm 2$ & $23 \pm 1$ \\
p41 & $21 \pm 1$ & $17 \pm 2$ & $19 \pm 4$ & $25 \pm 1$ \\
p24 & $52 \pm 3$ & $61 \pm 4$ & $41 \pm 3$ & $50 \pm 2$ \\
& V313A & K314A & N315A & W316A \\
Pr55 & $22 \pm 1$ & $31 \pm 2$ & $22 \pm 1$ & $23 \pm 1$ \\
p41 & $14 \pm 4$ & $22 \pm 7$ & $22 \pm 1$ & $9 \pm 3$ \\
p24 & $64 \pm 5$ & $46 \pm 5$ & $55 \pm 2$ & $68 \pm 3$ \\
& M317A & T318A & E319A & \\
Pr55 & $36 \pm 4$ & $24 \pm 1$ & $19 \pm 1$ & \\
p41 & $31 \pm 2$ & $13 \pm 1$ & $20 \pm 2$ & \\
p24 & $33 \pm 2$ & $63 \pm 1$ & $60 \pm 1$ & \\
\hline
\end{tabular}

Virus-associated Gag proteins were quantified by a densitometer as described in the legend to Figure 2B. Percentages of $\operatorname{Pr} 55^{g a g}, \mathrm{p} 41^{g a g}$, and $\mathrm{p} 24^{g a g}$ were obtained by dividing the individual density unit by the total Gag protein density units and multiplying by 100 . All were derived from at least three independent trials each

showed moderately reduced membrane affinity, with about $30 \%$ of Gag aggregates were unable to bind to membranes. Mutants V313A, K314A, and W316A also exhibited a slightly reduced membrane binding capacity, with $5-15 \%$ of the cytoplasmic Gag were membrane-free. These results suggest that some mutations in the region adjacent to the C-terminus of MHR can affect the membrane binding of Gag. We also performed indirect immunofluorescence experiments to determine the subcellular distribution of the mutants. Figure 5 shows that mutants Q311A (B), K314A (C), W316A (D), M317A (E), and $4310-323(F)$, which were defective in virus particle production, all exhibited a fluorescence staining pattern similar to that of wt (A), with a heterogenous cytoplasmic staining and perinuclear ring, suggesting that the mutations do not significantly affect the subcellular distribution of Gag.

The substantially reduced ability of the mutants to produce virions is probably due to a defect in Gag multimerization

Since most of the point mutations showed no significant effects on the membrane association and intracellular distribution of Gag, the diminished ability of the Gag mutants to produce virus particles may be due to a failure of the Gag molecules to undergo appropriate multimerization required for virus-like particle formation, as a consequence of impaired Gag-Gag interactions induced by the point mutations. To test this possibility, cell lysates containing Gag proteins were subjected to velocity sedimentation analysis by centrifugation through a $25-45 \%$ sucrose gradient. Gag mutants defective in oligomerization would be unable to form higher molecular-weight complexes required for particle formation, and thus they would remain in the lower sucrose density fractions. As shown in Figure 6, wt viruslike particles (VLP) and cytoplasmic Gag were recovered mostly in the high sucrose density fractions (fractions 3 and 4), which is consistent with a previous report [39]. The assembly-competent mutant E312A and the mutant E319A which produced virions at a level over $70 \%$ of that of wt, showed sedimentation of cytoplasmic Gag mostly in fractions 3 and 4 . In contrast, most of the mutants that were markedly defective in virus production (below $50 \%$ of the wt level), S310A, Q311A，V313A，K314A，W316A，M317A, and $\Delta 310-323$, could not undergo multimerization as well as the wt, as most of them were afloat in fractions 1 and 2. Although E312A and E319A had similar sedimentation pattern, the E312A produced much more virus particles than E319A. This suggests that E319A may have a defect in the process of virus budding though it can undergo multimerization efficiently. These data support the conclusion that the reduced efficiency of virus production in these mutants is mainly due to a defect in the process of multimerization of Gag into virus-like particles.

\section{Discussion}

We have demonstrated that removal of 14 amino acid residues adjacent to the $\mathrm{C}$-terminus of the HIV-1 CA major homology region (MHR) drastically reduces virus particle production. The HIV-1 capsid C-terminal domain (CTD), including the MHR and the adjacent downstream sequence, is required for capsid dimerization and has been referred to as the dimerization domain. The dimerization domain contains four $\alpha$-helices followed by a structurally disordered segment that consists of the last 12 or 13 residues of CA [28, 44, 50]. Although the CTD has been well known for its importance in HIV assembly, the extent of damage to virus particle production may vary depending 

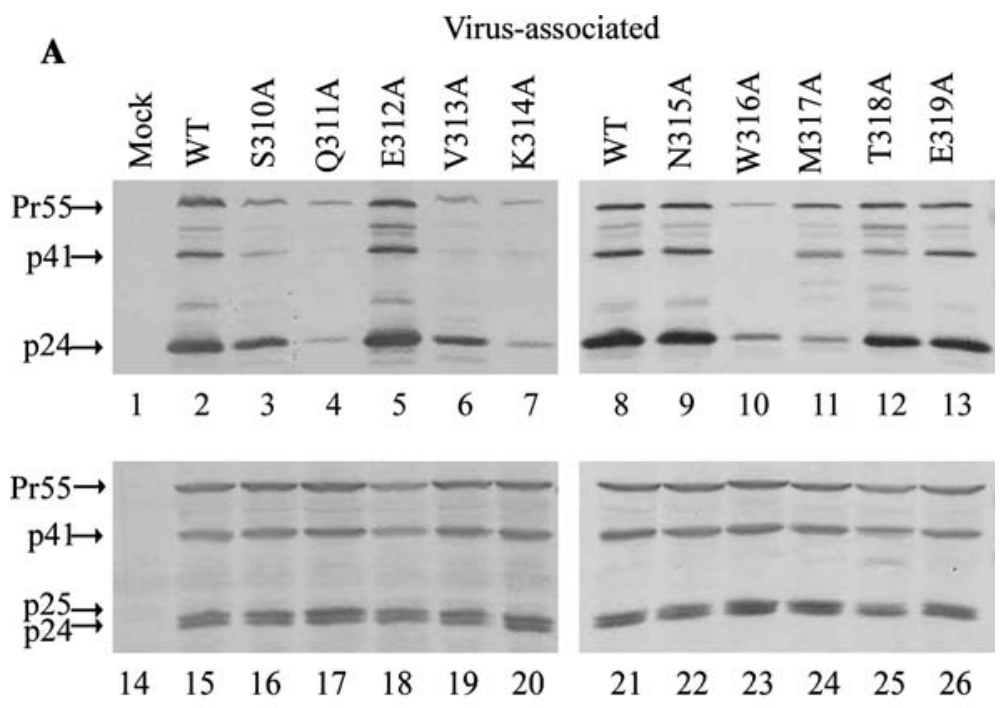

Cell lysates

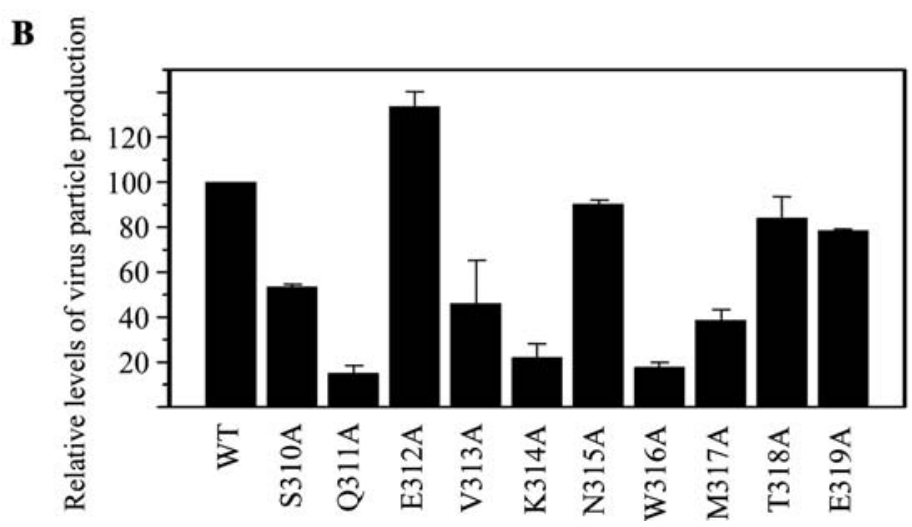

Figure 3. Effects of alanine substitutions on assembly and processing of HIV Gag proteins. (A) 293T cells were transfected with $20 \mu \mathrm{g}$ of the designated plasmids. At $48 \mathrm{~h}$ posttransfection, culture supernatants were collected, filtered, and pelleted through $20 \%$ sucrose cushions. Viral pellet samples (lanes 1-13) and cell lysate samples (lanes 14-26) were prepared for protein analysis as described in the legend to Figure 2. Membrane-bound HIV proteins were probed with a mouse anti-HIV-1 CA monoclonal antibody followed by a horse anti-mouse alkaline phosphatase-conjugated antibody as secondary antibody. Positions of the HIV Gag proteins Pr55, p41, p24/25, and p24 are indicated on the left. (B) Relative levels of virus-associated Gag proteins. Virus-associated Gag proteins in each sample were quantified by scanning p2 $4^{g a g}$-associated band densities on the immunoblot. Ratios of total Gag protein levels were calculated for each of the samples, and normalized to that of the wild type in parallel experiments. Error bars indicated standard deviations.

on the boundary of gag coding sequence that has been deleted. Deletion of the MHR or the region adjacent to MHR $(\Delta 310-323)$ seems to be more detrimental to virus production than the other three deletions $(\Delta 322-338, \Delta 339-365$, and $\Delta 368-378)$ located distal to the MHR. The severe defect of $\Delta 310-323$ in virus particle production is not surprising, as a number of studies have demonstrated the importance of the MHR in virus assembly $[27,38,40]$. Contrary to our results, an
HIV mutant lacking the MHR ( $\Delta 283-305)$ has previously been shown to be capable of producing virus particles at a level over $50 \%$ of that of wt [16]. This discrepancy could be due to our MHR deletion mutant $(\Delta 281-308)$ having a more extensive deletion that includes the sequence flanking the MHR. In addition, the possibility that the discrepancy can be attributed to the different systems employed cannot be excluded. The $\Delta 310-323$ mutant containing a replacement of 


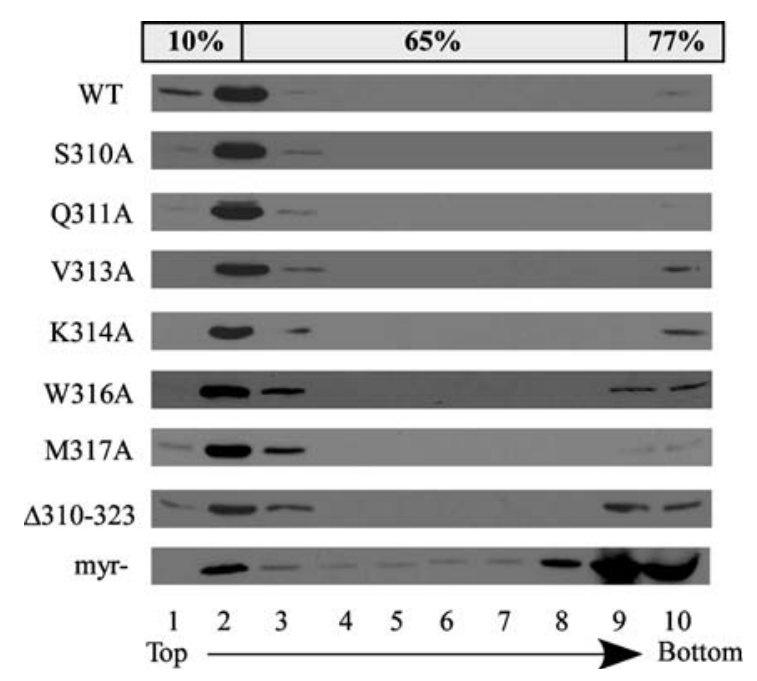

Figure 4. Membrane flotation analysis of HIV Pr5 $5^{\text {gag }}$ proteins. 293T cells were transfected with the PR-defective version of the wild type or designated mutants. Two days after transfection, the harvested cells were homogenized and the cell lysates were subjected to equilibrium flotation centrifugation analysis as described in Materials and methods. A total of 10 fractions were collected, from the top downwards. Aliquots of each fraction were resolved by SDS-PAGE (10\%), and probed with a monoclonal antibody directed against HIV-1 CA. During ultracentrifugation, membrane-bound Gag proteins floated to the $10-65 \%$ sucrose interface and became enriched in fraction 2. deleted sequence with two foreign residues, $\mathrm{R}$ and I, has the $\alpha$-helix 2 (residues 311-324) almost deleted. Crystallography studies have suggested that the CTD (residues 278-363) dimerizes by parallel packing of $\alpha$-helix 2 across the dimer interface [28]. Thus, removal of $\alpha$-helix 2 may disrupt Gag-Gag interaction and consequently abolish production of virus particles.

The disordered region at the C-terminal domain of CA contains a highly conserved glycinerich domain, 353-GVGGPG-358, and the residues 359-HKARVL-364 which constitutes the N-terminal portion of a putative $\alpha$-helix extending to SP1. Point mutations at these regions have been shown to markedly reduce particle production $[32,36,37$, $39,41]$. In addition, point mutations in SP1 impaired both membrane binding and multimerization of Gag [41]. However, either the $\Delta 339-365$, which lacks the disordered region, or the $\Delta 368$ 378 , does not eliminate virus particle production (Figure 2). To test whether this discrepancy is due to a difference in the system employed, we created a number of alanine substitution mutants H359A, K360A, and M368A, and compared the effects of these mutations on virus production with those of $\Delta 339-365$ and $\Delta 368-378$. We found that H359A
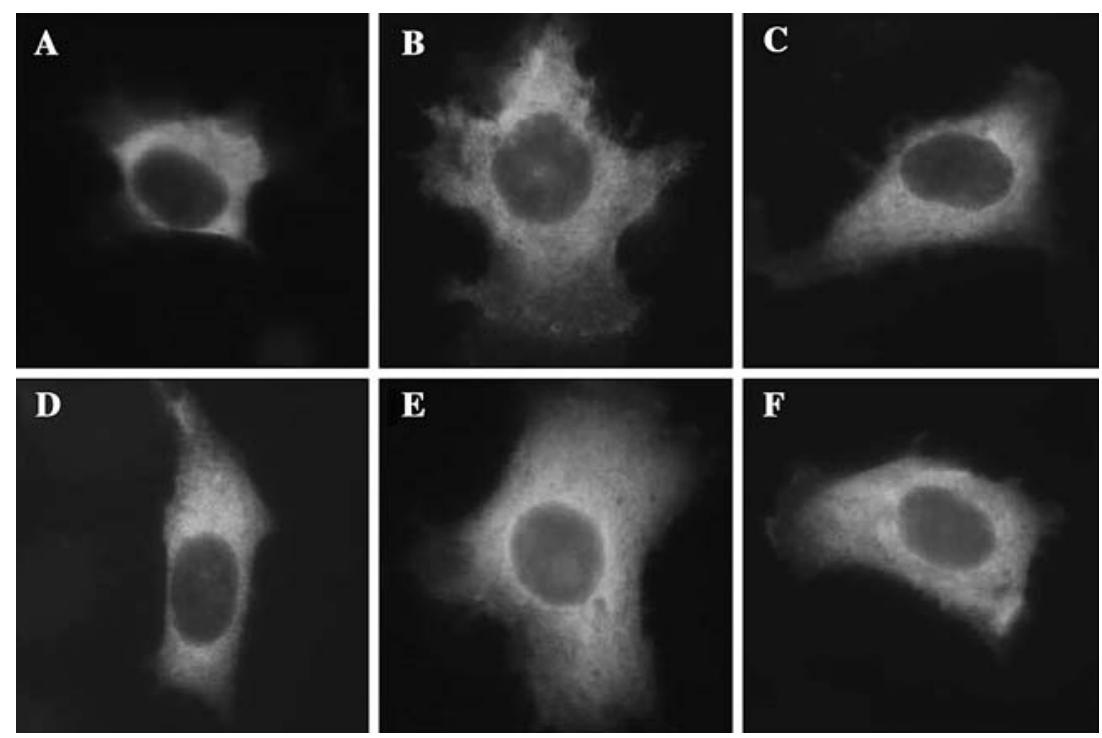

Figure 5. Detection of HIV Pr55 $5^{\text {gag }}$ proteins in HeLa cells by indirect immunofluorescence. HeLa cells grown in coverslips were transfected with the PR-defective version of the wild type (A) or mutants Q311A (B), K314A (C), W316A (D), M317A (E), and $\Delta 310-323$ (F). At $48 \mathrm{~h}$ posttransfection, the cells were fixed and permeabilized for immunofluorescence assays as described in Materials and methods. The primary antibody was a mouse monoclonal antibody directed against HIV-1 CA (1:500), and the secondary antibody was a 1:100 dilution of a rhodamine-conjugated rabbit anti-mouse antibody. Mock-transfected HeLa cells and cells not exposed to the primary antibody yielded no signals (data not shown). 
and M368A both diminished virus production profoundly at a level comparable to that of the same point mutations in previous reports [39, 42]. Compared with the deletion mutations $\Delta 339-365$ and $\Delta 368-378$, point mutations H359A and M368A appeared to cause more severe damage to virus production (data not shown). It is conceivable that potentially deleterious interactions induced by the point mutations may have been removed altogether in the $\Delta 339-365$ and $\Delta 368-378$ mutations.

Of the $\mathrm{N}$-terminal 10 amino acid residues deleted in $\Delta 310-323$ residues S310, E312, V313, W316, M317, and E319 have been proposed to lie in the $\mathrm{C}$-terminal dimer interface shown by crystallographic studies. It has been demonstrated previously that alanine substitutions at either residue W316 or M317, both located at the center of the dimer interface ( $\alpha$-helix 2), block CA dimerization in vitro [28] or markedly reduce virus particle production, while mutations E312A and Q324A, both located in the peripheral dimer interface, have no adverse effects on particle production [28, 30]. We observed similar results by alanine substitution at residues E312, W316, or M317 (Figure 3). However, in contrast to the E312A mutation, mutations Q311A and K314A markedly impair virus particle production, even though neither is located at the center of the $\alpha$ helix and neither belongs to the dimer interface residues [50]. Velocity sedimentation analysis of the intracellular Gag mutants indicated that there is good agreement between the degree of impairment in virus particle production and the extent of the defect in Gag multimerization (Figure 6). These data support the idea that the CA residues of $\alpha$-helix 2 play a role in facilitating Gag multimerization, possibly by promoting or stabilizing the Gag-Gag interactions. Residues Q311 and K314 may make a significant contribution to the stability of the dimer through hydrophobic interactions with the dimer interface residues. Thus, mutations in either residue could disrupt Gag multimerization by reducing the intermolecular Gag-Gag interaction, thus resulting in abolishment of virus particle production.

Mutations in the C-terminal disordered region of CA or in SP1 have been shown to impair both membrane binding and multimerization of Gag, and consequently, to reduce virus particle production [37, 41]. However, another research group [39]

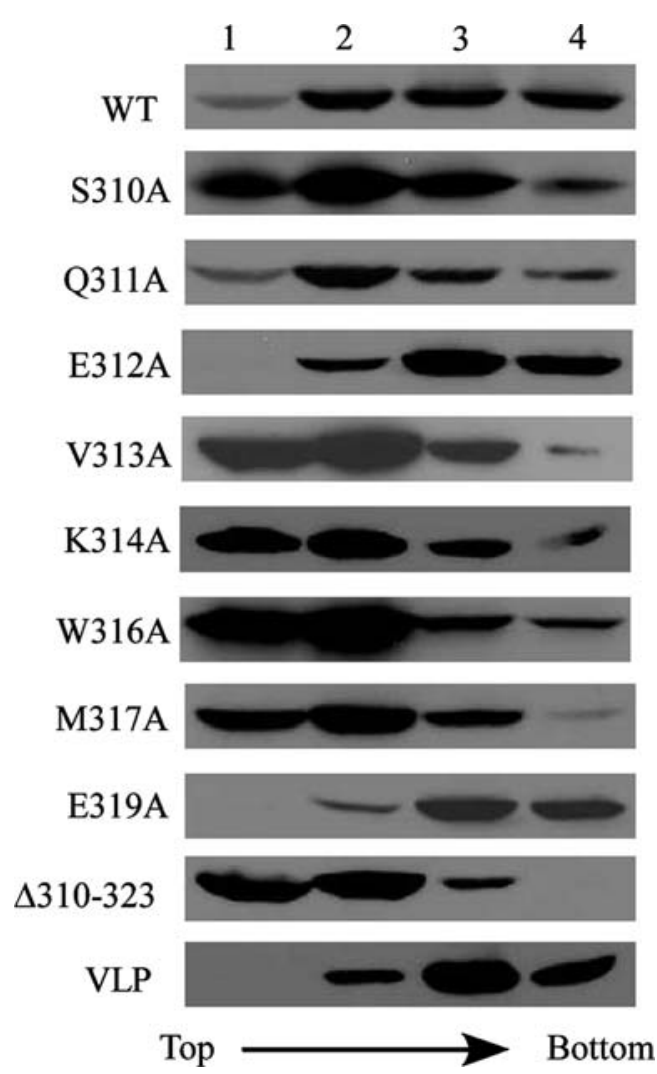

Figure 6. Velocity sedimentation analysis of cytoplasmic Gag precursor complexes. 293T cells were transfected with $20 \mu \mathrm{g}$ of the PR-defective version of the constructs indicated. Two days after transfection, the cells were homogenized and extracted cytoplasmic lysates were centrifuged through a 23, 35, and $45 \%$ sucrose step gradient at $130,000 \times g$ for $1 \mathrm{~h}$. As a control, 'VLP' (virus-like particle)-containing pellets derived from concentrated wild-type HIV Gag particles were centrifuged through the gradient in parallel. Fractions were collected from the top of the gradient. Aliquots of each fraction were subjected to SDS-PAGE $(10 \%)$, and probed with a monoclonal antibody directed against HIV-1 CA.

has demonstrated that the same C-terminal point mutations have no significant effect on the membrane-binding ability of Gag although, in agreement with the previous report, they are detrimental to both Gag multimerization and virus particle production. Electron microscopy revealed that rod-like structures protrude from cells that express the multimerization-defective mutants, suggesting a defective budding of the C-terminal CA mutants [39]. It remains to be determined whether mutations in $\alpha$-helix 2 have any effect on the process of virus budding. However, it is clear that point mutations either in the center or at the periphery of the dimer interface appears to have no great effect on the 
membrane binding and subcellular distribution of Gag, despite the fact that the mutations have affected Gag multimerization significantly (Figures 4-6). This supports the notion that strong Gag-Gag interaction may not be required for the efficient binding of HIV-1 Gag to membranes [51, 52]. However, there is a possibility that Gag multimerization may promote or stabilize the binding of Gag complexes to membranes under certain circumstances [53]. This may explain in part why the multimerization-defective $\Delta 310-323$ mutant had slightly reduced membrane binding capacity. In conclusion, our results suggest that residues situated in the $\alpha$-helix directly $\mathrm{C}$-terminal to the MHR are required for efficient Gag multimerization, a critical step in virus assembly during virus replication.

\section{Acknowledgements}

We thank H.-C. Chiu and W.-H. Liao for reagents and technical assistance. The hybridoma clone $183 \mathrm{H} 12-5 \mathrm{C}$ was provided by Bruce Chesebro of the AIDS Research and Reference Reagent Program, Division of AIDS, NIAID, MD. This work was supported by grants VGH93-309 from the Taipei Veterans General Hospital and NSC932320-B-010-015 from the National Science Council, Taiwan, Republic of China

\section{References}

1. Gelderblom H.R., Assembly and morphology of HIV: potential effect of structure on viral function. AIDS 5: 617638, 1991

2. Hunter E., Macromolecular interactions in the assembly of HIV and other retroviruses. Semin. Virol. 5: 71-83, 1994.

3. Wills J.W. and Craven R.C., Form, function, and use of retroviral gag proteins. AIDS 5: 639-654, 1991.

4. Henderson L.E., Bowers M.A., Sowder R.C. II, Serabyn S.A., Johnson D.G., Bess J.W. Jr, Arthur L.O., Bryant D.K. and Fenselau C., Gag proteins of the highly replicative MN strain of human immunodeficiency virus type 1: posttranslational modifications, proteolytic processing, and complete amino acid sequences. J. Virol. 66: 1856-1865, 1992.

5. Leis J., Baltimore D., Bishop J.B., Coffin J., Fleissner E., Goff S.P., Oroszlan S., Robinson H., Skalka A.M., Temin H.M. and Vogt V., Standardized and simplified nomenclature for proteins common to all retroviruses. J. Virol. 62: 1808-1809, 1988

6. Mervis R.J., Ahmad N., Lillehoj E.P., Raum M.G., Salazar F.H.R., Chan H.W. and Venkatesan V., The gag gene products of human immunodeficiency virus type 1: alignment within the gag open reading frame, identification of posttranslation modifications, and evidence for alternative gag precursors. J. Virol. 62: 3993-4002, 1988.

7. Swanstrom R. and Wills J.W., Synthesis, assembly, and processing of viral proteins. In: Coffin J.M., Hughes S.H. and Varmus H.E. (Eds), Retroviruses. Cold Spring Harbor Laboratory Press, Cold Spring Harbor, NY, 1997.

8. Chen Y.-L., Ts'ai P.-W., Yang C.-C. and Wang C.-T., Generation of infectious virus particles by transient coexpression of human immunodeficiency virus type $1 \mathrm{gag}$ mutants. J. Gen. Virol. 78: 2497-2501, 1997.

9. Gottlinger H.G., Sodroski J.G. and Haseltine W.A., Role of capsid precursor processing and myristylation in morphogenesis and infectivity of human immunodeficiency virus type1. Proc. Natl. Acad. Sci. USA 86: 5781-5785, 1989.

10. Kohl N.E., Emini E.A., Schleif W.E., Davis L.J., Heimbach J.C., Dixon R.A.F., Scolnick E.M. and Sigal I.S., Active human immunodeficiency virus protease is required for viral infectivity. Proc. Natl. Acad. Sci. USA 85: 4686-4690, 1988.

11. Peng C., Ho B.K., Chang T.W. and Chang N.T., Role of human immunodeficiency virus type 1 -specific protease in core particle maturation and viral infectivity. J. Virol. 63: 2550-2556, 1989.

12. Jacks T., Power M.D., Masiarz F.R., Luciw P.A., Barr P.J. and Varmus H.E., Characterization of ribosomal frameshifting in HIV-1 gag-pol expression. Nature 331: 280-283, 1988.

13. Chiu H.-C., Liao W.-H., Chen S.-W. and Wang C.-T., The human immunodeficiency virus type 1 carboxyl-terminal third of capsid sequence in Gag-Pol is essential but not sufficient for efficient incorporation of Pr160gag-pol into virus particles. J. Biomed. Sci. 11: 398-407, 2004.

14. Huang M. and Martin M.A., Incorporation of $\operatorname{Pr} 160^{\text {gag-pol }}$ into virus particles requires the presence of both the major homology region and adjacent C-terminal capsid sequences within the Gag-Pol polyprotein. J. Virol. 71: 4472-4478, 1997.

15. Smith A.J., N. Srinivasakumar N., Hammarskjold M.-L. and Rekosh D., Requirements for the incorporation of Pr160 ${ }^{\text {gag-pol }}$ from human immunodeficiency virus type 1 into virus-like particles. J. Virol. 67: 2266-2275, 1993.

16. Srinivasakumar N., Hammarskjold M.L. and Rekosh D., Characterization of deletion mutations in the capsid region of human immunodeficiency virus type 1 that affect particle formation and Gag-Pol precursor incorporation. J. Virol. 69: 6106-6114, 1995.

17. Freed E.O., HIV Gag proteins: diverse functions in the virus life cycle. Virology 251: 1-15, 1998.

18. Scarlata S. and Carter C., Role of HIV-1 Gag domains in viral assembly. Biochim. Biophys. Acta 1614: 62-72, 2003.

19. Bryant M. and Ratner L., Myristoylation-dependent replication and assembly of human immunodeficiency virus 1 . Proc. Natl. Acad. Sci. USA 87: 523-527, 1990.

20. Morikawa Y., Hockley D.J., Nermut M.V. and Jones I.M., Roles of matrix, p2, and N-terminal myristoylation in human immunodeficiency virus type 1 gag assembly. J. Virol. 74: 16-23, 2000.

21. Ono A. and Freed E.O., Binding of human immunodeficiency virus type 1 Gag to membrane: role of the matrix amino terminus. J. Virol. 73: 4136-4144, 1999.

22. Ono A., Demirov D. and Freed E.O., Relationship between human immunodeficiency virus type 1 Gag multimerization and membrane binding. J. Virol. 74: 5142-5150, 2000. 
23. Derdowski A., Ding L. and Spearman P., A novel fluorescence resonance energy transfer assay demonstrates that the human immunodeficiency virus type 1 Pr55Gag I domain mediates Gag-Gag interactions. J. Virol. 78: 12301242, 2004

24. Sandefur S., Varthakavi V. and Spearman P., The I domain is required for efficient plasma membrane binding of human immunodeficiency virus type 1 Pr55gag. J. Virol. 72: 2723-2732, 1998

25. Spearman P., Wang J.-J., Heyden N.V. and Ratner L., Identification of human immunodeficiency virus type $1 \mathrm{Gag}$ protein domains essential to membrane binding and particle assembly. J. Virol. 68: 3232-3242, 1994.

26. Zhang Y., Qian H., Love Z. and Barklis E., Analysis of the assembly functions of the human immunodeficiency virus type $1 \mathrm{Gag}$ protein nucleocapsid domain. J. Virol. 72: 1782 1789, 1998.

27. Dorfman T., Bukovsky A., Ohagen A., Hoglund S. and Gottlinger H.G., Functional domains of the capsid protein of human immunodeficiency virus type 1. J. Virol. 68: 8180-8187, 1994.

28. Gamble T.R., Yoo S., Vajdos F.F., von Schwedler U.K., Worthylake D.K., Wang H., McCutcheon J.P., Sundquist W.I. and Hill C.P., Structure of the carboxyl-terminal dimerization domain of the HIV-1 capsid protein. Science 278: 849-853, 1997.

29. Ganser-Pornillos B.K., von Schwedler U.K., Stray K.M., Aiken C. and Sundquist W.I., Assembly properties of the human immunodeficiency virus type 1 CA protein. J. Virol. 78: 2545-2552, 2004

30. von Schwedler U.K., Stray K.M., Garrus J.E. and Sundquist W.I., Functional surfaces of the human immunodeficiency virus type 1 capsid protein. J. Virol. 77: 5439-5450, 2003.

31. Freed E.O., Viral late domain. J. Virol. 76: 4679-4687, 2002.

32. Accola M.A., Hoglund S. and Gottlinger H.G., A putative $\alpha$-helical structure which overlaps the capsid-p2 boundary in the human immunodeficiency virus type 1 gag precursor is critical for viral particle assembly. J. Virol. 72: 20722078, 1998.

33. Borsetti A., Ohagen A. and Gottlinger H.G., The Cterminal half of the human immunodeficiency virus type 1 Gag precursor is sufficient for efficient particle assembly. J. Virol. 72: 9313-9317, 1998.

34. Wang C.-T., Lai H.-Y. and Li J.-J., Analysis of minimal human immunodeficiency virus type 1 gag coding sequences capable of virus-like particle assembly and release. J. Virol. 72: 7950-7959, 1998.

35. Accola M.A., Strack B. and Gottlinger H.G., Efficient particle production by minimal Gag constructs which retain the carboxy-terminal domain of human immunodeficiency virus type 1 capsid-p2 and a late assembly domain. J. Virol. 74: 5395-5402, 2000.

36. Abdurahman S., Hoglund S., Goobar-Larsson L. and Vahlne A., Selected amino acid substitutions in the Cterminal region of human immunodeficiency virus type 1 capsid protein affect virus assembly and release. J. Gen. Virol. 85: 2903-2913, 2004.

37. Liang C., Hu J., Whitney J.B., Kleiman L. and Wainberg M.A., A structurally disordered region at the $\mathrm{C}$ terminus of capsid plays essential roles in multimerization and membrane binding of the gag protein of human immunodeficiency virus type 1. J. Virol. 77: 1772-1783, 2003.

38. Mammano F., Ohagen A., Hoglund S. and Gottlinger H.G., Role of major homology region of human immuno- deficiency virus type 1 in virion morphogenesis. J. Virol. 68: 4927-4936, 1994.

39. Melamed D., Mark-Danieli M., Kenan-Eichler M., Kraus O., Castiel A., Laham N., Pupko T., Glaser F., Ben-Tal N. and Bacharach E., The conserved carboxy terminus of the capsid domain of human immunodeficiency virus type 1 Gag protein is important for virion assembly and release. J. Virol. 78: 9675-9688, 2004

40. Provitera P., Goff A., Harenberg A., Bouamar F., Carter C. and Scarlata S., Role of the major homology region in assembly of HIV-1 Gag. Biochemistry 40: 5565-5572, 2001.

41. Guo X., Roldan A., Hu J., Wainberg M.A. and Liang C., Mutation of the SP1 sequence impairs both multimerization and membrane-binding activities of human immunodeficiency virus type 1 Gag. J. Virol. 79: 1803-1812, 2005.

42. Liang C., Hu J., Russell R.S., Roldan A., Kleiman L. and Wainberg M.A., Characterization of a putative $\alpha$-helix across the capsid-spl boundary that is critical for the multimerization of human immunodeficiency virus type 1 Gag. J. Virol. 76: 11729-11737, 2002.

43. Chien A.-I., Liao W.-H., Yang D.-M. and Wang C.-T., A domain directly $\mathrm{C}$-terminal to the major homology region of human immunodeficiency type 1 capsid protein plays a crucial role in directing both virus assembly and incorporation of Gag-Pol. Virology 348: 84-95, 2006.

44. Momany C., Kovari L.C., Prongay A.J., Keller W., Gitti K., Lee B.M., Gorbalenya A.E., Tong L., McClure J., Ehrlich L.S., Summers M.F., Carter C. and Rossmann M.G., Crystal structure of dimeric HIV-1 capsid protein. Nat. Struct. Biol. 63: 763-770, 1996.

45. Page K.A., Landau N.R. and Littman D.R., Construction and use of a human immunodeficiency virus vector for analysis of virus infectivity. J. Virol. 64: 5270-5276, 1990.

46. Wang C.-T. and Barklis E., Assembly, processing, and infectivity of human immunodeficiency virus type $1 \mathrm{Gag}$ mutants. J. Virol. 67: 4264-4273, 1993.

47. Chiu H.-C., Yao S.-Y. and Wang C.-T., Coding sequences upstream of the human immunodeficiency virus type 1 reverse transcriptase domain in Gag-Pol are not essential for incorporation of the $\operatorname{Pr} 160^{\text {gag-pol }}$ into virus particles. J. Virol. 76: 3221-3231, 2002.

48. Graham R. and van der Eb A., A new technique for the assay of infectivity of human adenovirus 5 DNA. Virology 52: 456-467, 1973.

49. Ebbets-Reed D., Scarlata S. and Carter C.A., The major homology region of the HIV-1 gag precursor influences membrane affinity. Biochemistry 35: 14268-14275, 1996.

50. Worthylake D.K. Wang H., Yoo S., Sundquist W.I. and Hill C.P., Structures of the HIV-1 capsid protein dimerization domain at 2.6 A resolution. Acta Crystallogr D Biol Crystallogr 55: 85-92, 1999.

51. Lee Y.M., Liu B. and Yu X.F., Formation of virus assembly intermediate complexes in the cytoplasm by wildtype and assembly-defective mutant human immunodeficiency virus type 1 and their association with membranes. J. Virol. 73: 5654-5662, 1999.

52. Ono A., Orenstein J.M. and Freed E.O., Role of the Gag matrix domain in targeting human immunodeficiency virus type 1 assembly. J. Virol. 74: 2855-2866, 2000.

53. Lindwasser O.W. and Resh M.D., Multimerization of human immunodeficiency virus type 1 Gag promotes its localization to barges, raft-like membrane microdomains. J. Virol. 75: 7913-7924, 2001. 